\title{
The Pleasure Deficit: Revisiting the "Sexuality Connection" in Reproductive Health
}

In a seminal 1993 article, Ruth Dixon-Mueller questioned the reproductive health field's conceptualization of sexuality, arguing that it had treated intercourse as a sanitized, emotionally neutral act. ${ }^{1}$ If one were to learn about human sexuality by reading family planning research and program manuals, she suggested, one would have no idea that sex leads to great enjoyment-as well as pain-for human beings. She called for a more gender-sensitive approach to sexuality in research and programming, including greater attention to the ways in which women want to maximize sexual enjoyment and minimize sexual harm, and to how these desires influence their reproductive health behaviors. Such an approach-which Dixon-Mueller called establishing the "sexuality connection" in reproductive health-not only would garner a more accurate understanding of sexuality and sexual risk reduction, but also would acknowledge women as sexual agents rather than merely as sexual victims or as "targets" of contraceptive programs and HIV prevention efforts.

During the nearly 15 years since Dixon-Mueller's article was published, many important developments regarding sexuality have occurred within the family planning field. Most symbolically, the phrase "reproductive health" has been superseded by "sexual and reproductive health," and the terms "sexual health" and "sexual rights" increasingly appear in public health and human rights discourse. *2 In addition, the HIV/AIDS epidemic has highlighted the desperate need for better data on sexual behaviors and spurred collaborations between clinicians and social scientists who study sexuality. ${ }^{3}$ Thus, at least at first glance, the reproductive health field has opened its doors to deeper explorations of sexuality.

Threats to women's sexual and reproductive well-being have been especially well documented during the past 10-15 years. An impressive body of work reveals the ways in which women's sexual autonomy-and thus their pregnancy and disease prevention practices-are limited by gender inequalities at both individual and structural levels. At the individual level, gender-based violence, ${ }^{4-9}$ nonvolitional sex $^{10,11}$ and relationship power imbalances ${ }^{12,13}$ all have been associated with reduced sexual autonomy and thus greater vulnerability to unintended pregnancy, HIV and other STIs, and reproductive morbidity ${ }^{14}$ and mortality. At the structural level, the combination of poverty and gender inequality

*Sandfort and Ehrhardt argue that the invention of sildenafil citrate (Viagra) and the subsequent outpouring of clinical and psychological research on sexual function and dysfunction contributed to the rise of sexual health as a concept (source: Sandfort TGM and Ehrhardt A, Sexual health: a useful public health paradigm or a moral imperative? Archives of Sexual Behavior, 2004, 33(3):181-187). leads many women to exchange sex for money, clothing, gifts and other goods-yet another risk factor for HIV infection and other adverse reproductive health outcomes. ${ }^{15-17}$ This literature has significantly deepened our understanding of how experiencing sexual harm influences women's sexual and reproductive health and risk.

However, the ways in which the positive aspects of sexual experience contribute to women's sexual health and risk are little understood. Despite a few notable exceptions, ${ }^{18,19}$ the public health research community has largely failed to explore the factors that contribute to optimal sexual functioning for women or the ways in which sexual pleasureseeking (as opposed to love-seeking or money-seeking) influences women's risk for unintended pregnancy and disease. This "pleasure deficit" inspired a 2006 review in The Lancet, ${ }^{20}$ in which the authors called for the promotion of pleasure in HIV and other STI prevention programs, and warned that negative messages about sexuality can undermine, rather than promote, effective condom use.

Notably, the authors of the Lancet review suggested that acknowledgment and discussion of pleasure has been absent from all areas of HIV and other STI programming, and not just those pertaining to women. However, at least some research has focused on the ways in which the desire for pleasure motivates men to take sexual risks. For example, several studies have examined the role of pleasure in men's decisions to have anal intercourse with other men without using condoms ("barebacking"), ${ }^{21-23}$ and others have documented heterosexual men's lack of interest in using male condoms during vaginal sex because they diminish sexual pleasure. ${ }^{24-27}$ These studies provide some insight into the ways in which men's desires for sexual enjoyment shape their willingness to use male condoms. They also explore how cultural norms about masculinity, such as the social benefits for men of sexual conquest and virility, can influence men's pleasure-seeking. ${ }^{28-30}$ In stark contrast, relatively little research has examined women's pleasure-seeking and how it influences their sexual and contraceptive behaviors.

Below, we discuss in greater detail some examples of the "pleasure deficit" for women in sexual and reproductive health research and programs, and highlight areas for future research.

\section{RESEARCH AND PROGRAMMING Women and Male Condoms}

The public health approach to women and their partners' use of male condoms has evolved significantly since the beginning of the HIV/AIDS epidemic. Many public health
By Jenny A. Higgins and Jennifer $S$. Hirsch

Jenny A. Higgins is a postdoctoral research associate, Office of Population Research, Princeton University, New Jersey. Jennifer S. Hirsch is associate professor, Department of Sociomedical Sciences, Mailman School of Public Health, Columbia University, New York. 
programs seek to strengthen women's skills to negotiate with male partners for condom use. However, a large body of research suggests that gender inequality (particularly in the social and financial realms) makes it difficult-and sometimes impossible-for women to ensure condom use. ${ }^{13,31-34}$ Furthermore, even when women are able to negotiate for condom use, they may not want to do so, because some women view condoms as incompatible with sex that is intimate, loving and monogamous. ${ }^{35-39}$ Thus, women's social, emotional and financial dependence on both men ${ }^{13}$ and romantic relationships ${ }^{40}$ can make it difficult for them to encourage male partners to use condoms.

In comparison, women's sexual resistance to condoms has been relatively unexplored. Theorists within the HIV field have developed behavioral models that directly ${ }^{41}$ or indirectly ${ }^{42}$ acknowledge the role of pleasure for both partners in shaping uptake and use of male condoms. In particular, the AIDS Risk Reduction Model asserts that how condoms feel matters to both women and men. ${ }^{41}$ Certainly, the empirical literature suggests that many men do not like using condoms because they curtail sexual sensation. ${ }^{24-26}$ A 14-country study by the Joint United Nations Programme on HIV/AIDS found that men's most frequently reported reason for not using condoms was reduced sexual pleasure. ${ }^{27}$

In sharp contrast, researchers rarely consider the possibility (although there have been a few exceptions ${ }^{43,44}$ ) that condoms' effects on pleasure may alter women's preferences or use patterns. Yet in our own qualitative research on sexual pleasure and contraceptive use in the southeastern United States, we found that a greater proportion of women than of men disliked the feeling of male condoms. Some women reported that condoms "cover up" sensation and exacerbate vaginal dryness, which led them to discontinue use. ${ }^{45}$ Systematic research is critically needed to examine how the desire for sexual pleasure (or, more broadly, the full range of reasons why women have sex) shapes women's willingness to use male condoms.

\section{Research and Development}

Male condoms are not the only contraceptive method for which information on pleasure is lacking: Most contraceptive research and development has failed to collect information on how various methods influence women's sexual functioning and enjoyment. Information is particularly scarce for hormone-based methods. Although the effects of hormonal contraceptives on ovulation have been extensively documented, these contraceptives' potential effects on the increase in libido that women often experience during ovulation have received little attention. ${ }^{46,47}$ Nor have hormonal and other contraceptives' effects on sexual pleasure, and thus on contraceptive preferences and practices, been extensively studied, even during new product development. ${ }^{48}$ This lack of attention to the sexual side effects of hormone-based methods for women is particularly striking when viewed against the concern with side effects evident in acceptability studies of hormone-based methods under development for men. ${ }^{49-51}$

Fortunately, the sexual dimensions of acceptability have received more attention in the development of microbicides $^{52-57}$ and the female condom, ${ }^{58,59}$ both of which emerged from efforts to create female-controlled HIV prevention strategies (rather than from the family planning field). ${ }^{60}$ Ideally, all future contraceptive development and acceptability research will demonstrate the same concern for women's sexual functioning as hormonal trials have for the sexual functioning of men.

\section{Contraceptive Use Patterns}

A pleasure deficit also exists in most research exploring the ways in which women use-or fail to use-the contraceptive methods currently on the market. Few systematic reviews exist of these methods' effects on women's libido, enjoyment, lubrication or ability to achieve orgasm, or of how such effects shape the uptake, continuation and consistency of use. Similarly, current behavioral models of contraceptive decision making suggest that a woman's choice and consistent use of a particular method are related primarily to access, effectiveness, ease of use and the woman's desire to limit or space births; models rarely consider how methods either enhance or detract from the sexual experience.

Data do suggest that a woman's sexual experiences can shape contraceptive practices, and vice versa. Again, research on the female condom has been particularly innovative in this regard. A woman's sexual comfort with and enjoyment of this method (influenced by such factors as the polyurethane's enhancement of heat transfer and the potential for increased clitoral stimulation from the condom's outer ring) reportedly contribute to uptake and continuation. ${ }^{58,59,61-63}$

Other methods also have been studied for sexual acceptability, albeit less comprehensively. In a longitudinal study of new oral contraceptive users in the United States, researchers found that a decrease in users' libido and sexual enjoyment was strongly associated with discontinuation. ${ }^{48}$ A South African study of an injectable progestin contraceptive found that many women disliked the increase in vaginal wetness that they experienced while using this method, a factor that hindered uptake. ${ }^{64}$ A study of tubal ligation revealed that a large majority of women experienced no consistent change in sexual interest following the procedure, and those who reported changes were more likely to report an increase rather than a decrease in sexual interest and pleasure. ${ }^{65}$ In a study of the features most likely to shape contraceptive method choice, women ranked "lack of interference with sexual pleasure" as a "very important" contraceptive characteristic just as often as men did (30\% of men, $28 \%$ of women). ${ }^{66}$ Similarly, our qualitative data suggest that the way contraceptives alter "sexual aesthetics" (sensation, libido, lubrication, spontaneity and other sexual attributes) matters to women and men equally, and shapes both the choice of method and manner of use. ${ }^{45,67}$ However, only two participants out of 36 reported that their practitioners had ever asked them about 
their preferred sexual aesthetics, their sexual functioning (e.g., orgasm, vaginal lubrication) or other issues pertaining to sexuality (besides sexual risk).

Although these studies suggest that the uptake and continuation of contraception is influenced by how the methods make sex feel, few studies have examined multiple forms of contraception simultaneously, and even fewer have gone beyond individual experience to examine the cultural and social dimensions of what makes sex enjoyable. Future research should develop a model of the ways in which contraception can eroticize or de-eroticize sex for women; doing this requires a better understanding of how women experience their bodies when using contraceptives, including both how pleasure is enhanced and how discomfort (as defined uniquely in each cultural setting) is diminished, as well as how these experiences influence contraceptive practice. ${ }^{45}$ Guided by findings from preliminary qualitative studies in this area, quantitative fertility and reproductive surveys, such as the U.S. National Survey of Family Growth and the Demographic and Health Surveys, should systematically collect data on how various types of contraceptives affect participants' sexual experience. Only then will it be possible to determine statistically the degree to which pleasure-seeking predicts sexual risk behavior, experience of unintended pregnancy and transmission of HIV and other STIs.

However, attention to women's individual sexual experiences is not sufficient. These experiences should be linked to relational factors, such as power differentials within couples, and to social factors, such as gender inequality. For example, women may reject the use of male condoms because they diminish men's pleasure, which may be a critical part of the women's own sexual enjoyment; in contrast, men rarely express concern that methods for women detract from women's enjoyment. ${ }^{45}$ Building upon innovative scholarship on adolescent sexuality, ${ }^{43,68-70}$ researchers in this area must continue to explore the ways in which women's pleasure-seeking and contraceptive practices are shaped by their need for social affirmation and financial support from men.

\section{Unintended Pregnancy and Contraceptive Use}

Unintended pregnancy has long been of concern to policymakers and sexual and reproductive health practitioners. Worldwide, an estimated 80 million of the 210 million pregnancies each year (approximately 40\%) are unplanned, ${ }^{71}$ and the average woman will have at least one abortion in her lifetime. ${ }^{72}$ Most unintended pregnancies are caused by lack of contraceptive use, not by contraceptive failure. ${ }^{73,74}$ Current explanations for nonuse generally pertain either to women's knowledge of or access to contraceptive services (or lack thereof) or to gender-based power imbalances in sexual relationships, which can render women unwilling or unable to negotiate for contraceptive use with their male partners. ${ }^{75}$ Researchers have yet to suggest that unprotected sex may feel better to women or that the risk of pregnancy may heighten the sexual experience.
In contrast, the social science literature has explored some of the ways in which social constructions of pleasure motivate sexual behavior and HIV risk among men who have sex with men. ${ }^{76-78}$ Some scholars have demonstrated that barebacking has become eroticized in certain gay communities, even among men who understand the risk of HIV transmission. ${ }^{79}$ In some circumstances, social constructions of sexuality may mean that "riskier" sex is "hotter" sex. ${ }^{23}$

The family planning field stands to gain from similar examinations of the eroticization of pregnancy risk. One preliminary study found that an individual's or couple's temporary desire for a pregnancy during the heat of the sexual moment can lead to unprotected sex, even though a baby is not wholly desired (i.e., one or both partners are ambivalent about pregnancy). ${ }^{80}$ During the first author's tenure as an abortion clinic counselor, many clients described a temporary surrender to the fantasy of a pregnancy. ${ }^{81}$ Further, both women and men in our qualitative study indicated that the forging of intimacy between two people was an essential allure of sexual intercourse. ${ }^{45}$ In some instances, conception could become the ultimate extension of that closeness, even barring the desire for a pregnancy. ${ }^{82}$ We suggest that women and men may occasionally eroticize the possibility of a pregnancy with a particular partner, and that this desire could help explain their lack of contraceptive use.

The literature on HIV risk and barebacking provides a strong foundation for this idea, although the barebacking findings are not completely applicable to sex between women and men. Not only are the power dynamics different in heterosexual and same-sex couples, but the consequences of "risking" an unintended pregnancy differ from those of risking an HIV infection. Pregnancy, at least on some occasions, may be generative, life-affirming and relationship-strengthening. The field requires theoretical and empirical research on how the eroticization of pregnancy risk and the "heat of the moment" shape sexual risk practices, particularly if unintended pregnancy prevention remains an ongoing policy priority.

\section{FUTURE DIRECTIONS}

Despite the outpouring of HIV-related research on sexual behaviors in the last 10-15 years, the family planning field remains largely remiss in terms of Dixon-Mueller's "sexuality connection," specifically regarding the positive aspects of sexuality. However, there are certainly exceptions to this pleasure deficit.

Several family planning programs have already incorporated sexuality into their frameworks or have suggested such incorporation. ${ }^{83}$ (We should note that some of these programs are more than a decade old; surprisingly few have followed suit, providing another illustration of the pleasure

*Of course, pleasure-seeking is not the only factor that contributes to barebacking, which has also been associated with drug use (especially crystal methamphetamine), depression, social isolation and other factors (sources: references 21,22 and 80 ). 
deficit.) A Population Council program in Latin America and the Caribbean trained family planning counselors to discuss their clients' sexual relationships and practices before recommending certain methods over others and to review these issues during follow-up visits. ${ }^{84}$ Similarly, our research has led us to advocate for "pleasure profiling," in which a client's relationship and preferences for wetter, dryer, "natural" or more spontaneous sex are considered before contraceptive recommendations are made. ${ }^{45}$ For this approach to work optimally, culturally appropriate models of sexuality must be developed, implemented and evaluated, because women define and seek pleasure in different ways, both across and within cultural settings.

Along similar lines, offering sexuality training to health care providers more generally (and not just to family planning counselors) holds promise as a strategy to better meet women's and men's sexual health needs. Few clients report that health care practitioners ever ask them about sex, despite the evidence suggesting that such discussions-not merely those about sexual risk, but also about positive sexual experiences and satisfaction-yield significant benefits. ${ }^{85,86}$ Although most clients would welcome such dialogues, ${ }^{86}$ few feel comfortable initiating them on their own. In turn, providers often feel uncomfortable discussing sex with their clients or feel ill equipped to have such conversations. ${ }^{87}$ In response, one team of researchers has proposed a series of questions that clinicians may wish to ask clients as part of a proactive sexual health history, ${ }^{85}$ and others have produced alternative sexuality guidelines and training programs to support both providers and clients. ${ }^{88-91}$

Other auspicious approaches focus on the client level, rather than on the provider level. The Pleasure Project (<www.the-pleasure-project.org>), a United Kingdom-based nonprofit organization, aims to "put the sexy back into safer sex" by promoting the use of sex toys and sexual techniques that eroticize condom use (e.g., applying a condom to a partner's penis with one's mouth). In addition to its flashy, userfriendly Web site, the project generates support among public health practitioners at venues such as the International AIDS Conference and sponsors academic research in this area. Time will tell if such initiatives will be fundable and feasible, especially in the current political climate.

\section{CHALLENGES, CAUTIONS AND CONCLUSIONS}

We have highlighted a few initiatives that may serve as useful models for family planning practitioners. Yet, as the Lancet review notes, the few programs that have attempted to "promote protection and pleasure" have been small, unevaluated or based on anecdotal evidence. ${ }^{20}$ Furthermore, even if proven effective through evaluation, microlevel behavioral interventions are limited in their ability to change culture or social structure. ${ }^{3}$ For example, the programs described above can do little to address the broad social and cultural forces that make pleasure-seeking easier and more important for men than for women, or that limit women's sexual enjoyment by fueling gender-based sexual and physical violence. Similarly, systemic change will be required to alter the troubling assumptions about sexuality and reproduction that are alive and well within our field-for example, the belief that pregnancy prevention is primarily, if not entirely, women's responsibility, ${ }^{92}$ or the notion that sexual pleasure is irrelevant to women, especially in developing countries.

Another challenge to future work in this area is that pleasure-based initiatives may inadvertently perpetuate gender inequality. For example, some authors have cautioned against the promotion of a gender-neutral "right to sexual pleasure" as a basic human right, arguing that men's demands for sexual pleasure can infringe on women's human rights. ${ }^{29}$ Globally, men have more access to sexual enjoyment and autonomy than women do, ${ }^{13}$ and men's pleasure is likely to take precedence over women's pleasure, ${ }^{27}$ potentially leading to the abandonment of male condom use or to the eroticization of men's sexual domination.

Well-intentioned sexuality programs could also enforce existing unequal gender norms. For example, we have heard about sex technique workshops for married women in countries in which men's extramarital affairs fuel the spread of HIV. The basic premise of these programs is that greater sexual knowledge and comfort could discourage men's infidelity and thus reduce the risk of HIV transmission. Undoubtedly, many women could benefit from workshops in which they gain familiarity and comfort with their sexual selves. At the same time, however, such programs may fail to address issues such as men's abuses of their male privilege and their greater access to opportunities for extramarital sex.

Another example of a program that both reflects and perpetuates existing gender roles is one that promotes the use of male condoms by highlighting the advantages of delayed ejaculation. In India, the Kohinoor Xtra Time brand of condom is coated with a lubricant containing local anesthetic, which its promoters say enables longer lasting, and thus more pleasurable, intercourse. ${ }^{20}$ Such an advertising campaign could unintentionally reinforce pressure on men to fulfill expectations (their own or others') as sexual performers. Performance anxiety contributes to men's sexual dysfunction, including erectile difficulties, ${ }^{93}$ which in turn have been associated with condom misuse and nonuse. ${ }^{94-96}$ Both this example and that of the sex technique programs underscore the advantages and disadvantages of working within dominant constructions of masculinity to promote sexual and reproductive health. ${ }^{97,98}$ Furthermore, evidence on the average amount of time that women, either in India or in most other cultural settings, want to engage in vaginal intercourse is limited. More research on both women's and men's sexual preferences and needs would be warranted before large-scale condom campaigns aimed at lengthening pleasure are initiated.

We hope that any future work in this area will explore pleasure in relation to gender and power. A thorough understanding of pleasure-seeking behaviors requires consciousness both of social inequality and of cross-cultural and intracultural differences in the ways in which women 
and men seek pleasure. Future research should develop models to capture the various aspects of pleasure, and explore how masculinity and femininity shape these pleasures in each research setting.

Despite these challenges, we have tried to suggest the importance of pleasure-seeking to sexual and reproductive health. Researchers and program developers can no longer assume that the sexual aspects of family planning are irrelevant to women. Rather, they should work from the notion that the way sex feels matters to women, and that recognizing and addressing this aspect of women's lives will positively influence sexual risk behaviors-and more broadly, their sexual health and well-being.

\section{REFERENCES}

1. Dixon-Mueller R, The sexuality connection in reproductive health, Studies in Family Planning, 1993, 24(5):269-282.

2. Edwards WM and Coleman E, Defining sexual health: a descriptive overview, Archives of Sexual Behavior, 2004, 33(3):189-195.

3. Parker RG, Sexuality, culture, and power in HIV/AIDS research, Annual Review of Anthropology, 2001, Vol. 30, pp. 163-179.

4. McCarraher DR, Martin SL and Bailey PE, The influence of methodrelated partner violence on covert pill use and pill discontinuation among women living in La Paz, El Alto and Santa Cruz, Bolivia, Journal of Biosocial Science, 2006, 38(2):169-186.

5. Pallitto CC and O'Campo P, The relationship between intimate partner violence and unintended pregnancy: analysis of a national sample from Colombia, International Family Planning Perspectives, 2004, 30(4):165-173.

6. Watts $C$ and Mayhew $S$, Reproductive health services and intimate partner violence: shaping a pragmatic response in Sub-Saharan Africa, International Family Planning Perspectives, 2004, 30(4):207-213.

7. Stephenson R, Koenig MA and Ahmed S, Domestic violence and contraceptive adoption in Uttar Pradesh, India, Studies in Family Planning, 2006, 37(2):75-86.

8. El-Bassel $\mathrm{N}$ et al., HIV and intimate partner violence among methadone-maintained women in New York City, Social Science \& Medicine, 2005, 61(1):171-183.

9. Jewkes R et al., Factors associated with HIV sero-status in young rural South African women: connections between intimate partner violence and HIV, International Journal of Epidemiology, 2006, 35(6):1461-1468.

10. Kalmuss D, Nonvolitional sex and sexual health, Archives of Sexual Behavior, 2004, 33(3):197-209.

11. Doyal L, What Makes Women Sick: Gender and the Political Economy of Health, New Brunswick, NJ, USA: Rutgers University Press, 1995.

12. Pulerwitz J et al., Relationship power, condom use and HIV risk among women in the USA, AIDS Care, 2002, 14(6):789-800.

13. Blanc AK, The effect of power in sexual relationships on sexual and reproductive health: an examination of the evidence, Studies in Family Planning, 2001, 32(3): 189-213.

14. Finkler K, Women in Pain: Gender and Morbidity in Mexico, Philadelphia, PA, USA: University of Pennsylvania Press, 1994.

15. Farmer P, Connors M and Simmons J, Women, Poverty, and AIDS: Sex, Drugs, and Structural Violence, Monroe, ME, USA: Common Courage Press, 1996.

16. Preston-Whyte E et al., Survival sex and HIV/AIDS in an African city, in: Parker RG, Barbosa RM and Aggleton P, eds., Framing the Sexual Subject: The Politics of Gender, Sexuality, and Power, Berkeley, CA, USA: University of California Press, 2000, pp. 165-190.

17. Farmer P, AIDS and Accusation: Haiti and the Geography of Blame, Berkeley, CA, USA: University of California Press, 1992.

18. Moore K and Helzner JF, What's Sex Got to Do with It? Challenges for Incorporating Sexuality into Family Planning Programs, New York:
Population Council, 1996.

19. Zeidenstein S and Moore K, Learning About Sexuality: A Practical Beginning, New York: Population Council and International Women's Health Coalition, 1996.

20. Philpott A, Knerr W and Maher D, Promoting protection and pleasure: amplifying the effectiveness of barriers against sexually transmitted infections and pregnancy, Lancet, 2006, 368(9551):2028-2031.

21. Carballo-Diéguez A and Bauermeister J, "Barebacking": intentional condomless anal sex in HIV-risk contexts. Reasons for and against it, Journal of Homosexuality, 2004, 47(1):1-16.

22. Shernoff M, Without Condoms: Unprotected Sex, Gay Men, and Barebacking, New York: Routledge, 2005.

23. Junge B, Bareback sex, risk, and eroticism: anthropological themes (re-)surfacing in the post-AIDS era, in: Lewin E and Leap WL, eds., Out in Theory: The Emergence of Lesbian and Gay Anthropology, Chicago, IL, USA: University of Illinois Press, 2002, pp. 186-221.

24. Crosby RA et al., If the condom fits, wear it: a qualitative study of young African-American men, Sexually Transmitted Infections, 2004, 80(4):306-309.

25. Thomsen S, Stalker M and Toroitich-Ruto C, Fifty ways to leave your rubber: how men in Mombasa rationalise unsafe sex, Sexually Transmitted Infections, 2004, 80(6):430-434.

26. Khan SI et al., Safer sex or pleasurable sex? rethinking condom use in the AIDS era, Sexual Health, 2004, 1(4):217-225.

27. Joint United Nations Programme on HIV/AIDS (UNAIDS), Men and AIDS-A Gendered Approach, Geneva: UNAIDS, 2000

28. Pateman C, The Sexual Contract, Cambridge, MA, USA: Polity Press, 1988.

29. Oriel J, Sexual pleasure as a human right: harmful or helpful to women in the context of HIV/AIDS? Women's Studies International Forum, 2005, 28(5):392-404.

30. Bourgois PI, In Search of Respect: Selling Crack in El Barrio, Cambridge, UK: Cambridge University Press, 1995.

31. Amaro H, Raj A and Reed E, Women's sexual health: the need for feminist analyses in public health in the decade of behavior, Psychology of Women Quarterly, 2001, 25(4):324-334.

32. Ehrhardt AA and Exner TM, The impact of HIV infection on women's sexuality and gender role, in: Blumenthal SJ, Eichler A and Weissman G, eds., Women and AIDS: Promoting Healthy Behaviors, Rockville, MD, USA: U.S. Department of Health and Human Services, 1991, pp. 36-40.

33. Worth D, Sexual decision-making and AIDS: why condom promotion among vulnerable women is likely to fail, Studies in Family Planning, 1989, 20(6):297-307.

34. Exner TM et al., Beyond the male condom: the evolution of gender-specific HIV interventions for women, Annual Review of Sex Research, 2003, Vol. 14, pp. 114-136.

35. Sobo EJ, Inner-city women and AIDS: the psycho-social benefits of unsafe sex, Culture, Medicine \& Psychiatry, 1993, 17(4):455-485.

36. Sobo EJ, Finance, romance, social support, and condom use among impoverished inner-city women, Human Organization, 1995, 54(2):115128.

37. Hirsch JS, A Courtship After Marriage: Sexuality and Love in Mexican Transnational Families, Berkeley, CA, USA: University of California Press, 2003.

38. Hirsch JS et al., The social constructions of sexuality: marital infidelity and sexually transmitted disease-HIV risk in a Mexican migrant community, American Journal of Public Health, 2002, 92(8):1227-1237.

39. Knodel J and Pramualratana A, Prospects for increased condom use within marriage in Thailand, International Family Planning Perspectives, 1996, 22(3):97-102.

40. Cancian FM, The feminization of love, Signs: Journal of Women in Culture and Society, 1986, 11(4):692-709.

41. Catania JA, Kegeles SM and Coates TJ, Towards an understanding of risk behavior: an AIDS risk reduction model (ARRM), Health Education 
Quarterly, 1990, 17(1):53-72.

42. Montaño DE and Kasprzyk D, The theory of reasoned action and theory of planned behavior, in: Glanz K, Rimer BK and Lewis FM, eds. Health Behavior and Health Education: Theory, Research, and Practice, third ed., San Francisco, CA, USA: Jossey-Bass, 2002, pp. 67-98.

43. Holland J et al., The Male in the Head: Young People, Heterosexuality and Power, London: Turfnell Press, 1998.

44. Ehrhardt AA et al., HIV/STD risk and sexual strategies among women family planning clients in New York: Project FIO, AIDS and Behavior, 2002, 6(1):1-13.

45. Higgins JA and Hirsch JS, Pleasure and power: incorporating sexuality, agency, and inequality into research on contraceptive use and unintended pregnancy, American Journal of Public Health, 2008 (forthcoming).

46. Anderson-Hunt M et al., Hormones and female sexuality: developing a method for research, in: Zeidenstein S and Moore K, Learning About Sexuality: A Practical Beginning, New York: Population Council and International Women's Health Coalition, 1996, pp. 260-277.

47. Dennersetin L, Female sexuality, the menstrual cycle, and the pill, in: Zeidenstein S and Moore K, Learning About Sexuality: A Practical Beginning, New York: Population Council and International Women's Health Coalition, 1996, pp. 253-259.

48. Sanders SA et al., A prospective study of the effects of oral contraceptives on sexuality and well-being and their relationship to discontinuation, Contraception, 2001, 64(1):51-58.

49. Oudshoorn N, The Male Pill: A Biography of a Technology in the Making, Durham, NC, USA: Duke University Press, 2003.

50. Solomon H, Yount KM and Mbizvo MT, "A shot of his own": the acceptability of a male hormonal contraceptive in Indonesia, Culture, Health E Sexuality, 2007, 9(1):1-14

51. Robbins A, The effect of hormones on male sexuality: findings from clinical trials on male contraception, in: Zeidenstein S and Moore K, Learning About Sexuality: A Practical Beginning, New York: Population Council and International Women's Health Coalition, 1996, pp. 278297.

52. Bentley ME et al., Acceptability of a microbicide among women and their partners in a 4-country phase I trial, American Journal of Public Health, 2004, 94(7):1159-1164

53. Mantell JE et al., Microbicide acceptability research: current approaches and future directions, Social Science \& Medicine, 2005 60(2):319-330

54. Gross M, HIV and women: when words speak louder than actions, American Journal of Public Health, 2004, 94(7):1079-1080.

55. Severy LJ et al., A framework for examining the sustained acceptability of microbicides, AIDS and Behavior, 2005, 9(1):121-131.

56. Penman-Aguilar A et al., Integrating qualitative data into quantitative models of behavior: a mixed-method approach within applications of microbicide research, paper presented at the Microbicides 2006 Conference, Cape Town, South Africa, Apr. 23-26, 2006.

57. Pool Ret al., Ambivalence, sexual pleasure and the acceptability of microbicidal products in south-west Uganda, AIDS, 2000, 14(13):20582059.

58. Neilands TB and Choi KH, A validation and reduced form of the Female Condom Attitudes Scale, AIDS Education and Prevention, 2002 14(2):158-171

59. Artz $L$ et al., Effectiveness of an intervention promoting the female condom to patients at sexually transmitted disease clinics, American Journal of Public Health, 2000, 90(2):237-244.

60. Kaler A, The female condom in North America: selling the technology of "empowerment," Journal of Gender Studies, 2004, 13(2):139152.

61. Mantell JE et al., The acceptability of the female condom: perspectives of family planning providers in New York City, South Africa, and Nigeria, Journal of Urban Health, 2001, 78(4):658-668.

62. Penman-Aguilar A et al., Presenting the female condom to men: a dyadic analysis of effect of the woman's approach, Women $\&$ Health, 2002, 35(1):37-51.

63. Telles Dias PR, Souto K and Page-Shafer K, Long-term female condom use among vulnerable populations in Brazil, AIDS and Behavior, 2006, 10(Suppl. 4):S67-S75.

64. Smit J et al., Vaginal wetness: an underestimated problem experienced by progestogen injectable contraceptive users in South Africa, Social Science \& Medicine, 2002, 55(9):1511-1522.

65. Costello $C$ et al., The effect of interval tubal sterilization on sexual interest and pleasure, Obstetrics \& Gynecology, 2002, 100(3):511-517.

66. Grady WR, Klepinger DH and Nelson-Wally A, Contraceptive characteristics: the perceptions and priorities of men and women, Family Planning Perspectives, 1999, 31(4):168-175

67. Higgins J, Sexy feminisms and sexual health: theorizing heterosex, pleasure, and constraint for a public health research agenda, Atlantis, 2007, 31(2):72-81.

68. Carpenter LM, Virginity Lost: An Intimate Portrait of First Sexual Experiences, New York: New York University Press, 2005.

69. Tolman DL, Dilemmas of Desire: Teenage Girls Talk About Sexuality, Cambridge, MA, USA: Harvard University Press, 2002.

70. Phillips LM, Flirting with Danger: Young Women's Reflections on Sexuality and Domination, New York: New York University Press, 2000.

71. World Health Organization (WHO), Unsafe Abortion: Global and Regional Estimates of the Incidence of Unsafe Abortion and Associated Mortality in 2000, fourth ed., Geneva: WHO, 2004.

72. Alan Guttmacher Institute (AGI), Sharing Responsibility: Women, Society and Abortion Worldwide, New York: AGI, 1999.

73. Henshaw SK, Unintended pregnancy in the United States, Family Planning Perspectives, 1998, 30(1):24-29 \& 46.

74. Trussell J, Vaughan B and Stanford JB, Are all contraceptive failures unintended pregnancies? evidence from the 1995 National Survey of Family Growth, Family Planning Perspectives, 1999, 31(5):246-247 \& 260.

75. Brown SS and Eisenberg L, eds., The Best Intentions: Unintended Pregnancy and the Well-Being of Children and Families, Washington, DC: National Academy Press, 1995

76. Dowsett GW, Practicing Desire: Homosexual Sex in the Era of AIDS, Stanford, CA, USA: Stanford University Press, 1996.

77. Parker RG, Beneath the Equator: Cultures of Desire, Male Homosexuality, and Emerging Gay Communities in Brazil, New York: Routledge, 1998.

78. Parker RG, Bodies, Pleasures, and Passions: Sexual Culture in Contemporary Brazil, Boston, MA, USA: Beacon Press, 1991.

79. Diaz RM, Trips to Fantasy Island: contexts of risky sex for San Francisco gay men, Sexualities, 1999, 2(1):89-112.

80. Peacock $\mathrm{N}$ et al., Exploring the complexities of pregnancy intentions for African American women, paper presented at the annual meeting of the American Public Health Association, Atlanta, GA, USA, Oct. 21-25, 2001.

81. Higgins J, Sex, unintended pregnancy, and poverty: one woman's evolution from "choice" to "reproductive justice," in: Jacob K, ed., Abortion Under Attack: Women on the Challenges Facing Choice, Seattle, WA, USA: Seal Press, 2006, pp. 31-42.

82. Higgins J, The pleasure deficit: the role of desire in contraceptive use, unpublished dissertation, Atlanta, GA, USA: Emory University, 2005.

83. Gordon $G$, Sexual reality: the gap between family planning services and clients' needs, in: Zeidenstein S and Moore K, Learning About Sexuality: A Practical Beginning, New York: Population Council and International Women's Health Coalition, 1996, pp. 363-379.

84. Becker J and Leitman E, Introducing sexuality within family planning: the experience of three HIV/STD prevention projects from Latin America and the Caribbean, Quality/Calidad/Qualité, New York: Population Council, 1997, No. 8.

85. Nusbaum MRH and Hamilton CD, The proactive sexual health his- 
tory, American Family Physician, 2002, 66(9):1705-1712.

86. Nusbaum MRH, Gamble GR and Pathman DE, Seeking medical help for sexual concerns: frequency, barriers, and missed opportunities, Journal of Family Practice, 2002, 51(8):706, <http://www. jfponline.com/pdf\%2F5108\%2F5108JFP_JFPOnline2.pdf>, accessed June 26, 2007.

87. Warner PH, Rowe T and Whipple B, Shedding light on the sexual history, American Journal of Nursing, 1999, 99(6):34-40.

88. Sadovsky R and Nusbaum MRH, Sexual health inquiry and support is a primary care priority, Journal of Sexual Medicine, 2006, 3(1):3-11.

89. Rosen R et al., Sexual communication skills in residency training: the Robert Wood Johnson model, Journal of Sexual Medicine, 2006, 3(1):37-46.

90. Taylor B and Davis S, Using the extended PLISSIT model to address sexual healthcare needs, Nursing Standard, 2006, 21(11):35-40.

91. Wimberly Y and Moore S, Sexual history taking should be taught in medical school, letter to the editor, American Family Physician, 2003, 68(2):223

92. Watkins SC, If all we knew about women was what we read in Demography, what would we know? Demography, 1993, 30(4):551-577.

93. Laumann EO et al., The Social Organization of Sexuality: Sexual Practices in the United States, Chicago, IL, USA: University of Chicago Press, 1994.
94. Crosby RA et al., Condom use errors and problems among college men, Sexually Transmitted Diseases, 2002, 29(9):552-557.

95. Sanders SA et al., Condom use errors and problems among young women who put condoms on their male partners, Journal of the American Medical Women's Association, 2003, 58(2):95-98.

96. Crosby R et al., Condom-use errors and problems: a neglected aspect of studies assessing condom effectiveness, American Journal of Preventive Medicine, 2003, 24(4):367-370

97. Mantell JE et al., The promises and limitations of female-initiated methods of HIV/STI protection, Social Science \& Medicine, 2006 63(8):1998-2009.

98. Gupta GR, Gender, sexuality and HIV/AIDS: the what, the why and the how, HIV/AIDS Policy \& Law Review, 2000, 5(4):86-93.

\section{Acknowledgments}

At the time this article was written, Jenny Higgins was supported by a National Institute of Mental Health training grant (T32 MH19139) and by a National Institute of Mental Health grant to the HIV Center for Clinical and Behavior Studies (P30 MH43250). The authors thank the following colleagues for their insights on earlier drafts of this article: Susie Hoffman, Shari Dworkin, Theresa Exner, Joanne Mantell, Theo Sandfort and Bobby Gillespie.

Author contact: jennyh@princeton.edu 